\title{
Références bibliographiques du dossier « la formation ouverte et à distance »
}

\section{Marie Lakermance}

\section{(2) OpenEdition}

\section{Journals}

Édition électronique

URL : https://journals.openedition.org/ries/1742

DOI : $10.4000 /$ ries. 1742

ISSN : 2261-4265

Éditeur

France Education international

Édition imprimée

Date de publication : 1 décembre 2003

Pagination : 149-153

ISBN : 978-2-8542-0558-9

ISSN : $1254-4590$

Référence électronique

Marie Lakermance, "Références bibliographiques du dossier « la formation ouverte et à distance » », Revue internationale d'éducation de Sèvres [En ligne], 34 I décembre 2003, mis en ligne le 23 septembre 2002, consulté le 07 juillet 2021. URL : http://journals.openedition.org/ries/1742 ; DOI : https://doi.org/ $10.4000 /$ ries. 1742

Ce document a été généré automatiquement le 7 juillet 2021

(C) Tous droits réservés 


\title{
Références bibliographiques du dossier « la formation ouverte et à distance »
}

\author{
Marie Lakermance
}

\section{Généralités}

1 BARBARANT Jean-Claude (réd.), Enseignement à distance : réalités, enjeux et perspectives, Conseil économique et social/Paris, 1997, 205 p.

Ce rapport dresse un constat pessimiste sur le fonctionnement de terrain et sur l'absence de prise en compte par l'état de l'importance des enjeux socio-économiques en la matière. Ses recommandations sont les suivantes: connaître et reconnaître l'existant; rationaliser les offres éparses des opérateurs; organiser la coopération de l'enseignement présentiel et de l'enseignement à distance en formation initiale; favoriser la création de produits éducatifs multimédias; exploiter pleinement les infrastructures et les améliorer.

2 Formations ouvertes multiressources, Cahiers d'études du CUEEP, $\mathrm{n}^{\circ}$ 28, 1995/02, $188 \mathrm{p}$.

Le concept de «formations ouvertes multiressources» vise à intégrer dans un même dispositif global différents modèles en faisant coopérer des séquences de formation à distance, des séquences de formation individualisée, des séquences de formation collective, et en mobilisant des ressources éducatives multiples à la fois humaines et matérielles. Cette université d'été entendait transférer les premiers résultats des recherches-actions, capitaliser différentes expériences et initialiser des dispositifs de formations ouvertes.

3 HARASIM Linda, HILTZ Starr Roxanne, TELES Lucio, TUROFF Murray, Learning networks: a field guide to teaching and learning online, MIT press/Cambridge/États-Unis, 1997, 329 p., bibliogr., index.

La première partie constitue une introduction sur les réseaux d'apprentissage à distance, leurs utilisateurs, les types de cours en ligne, les bénéfices pour les 
apprenants et les enseignants. Elle fait part d'exemples et d'expériences réalisés à différents niveaux de l'enseignement. La seconde partie traite des problèmes de conception et de mise en œuvre de programmes d'enseignement à distance, du rôle des enseignants et des apprenants et des écueils éventuels auxquels il faut s'attendre. La dernière partie propose une réflexion sur les nouvelles technologies comme nouveau paradigme pour l'éducation future.

HARRY Keith (éd.), Open and distance learning: a sélective bibliography for higher education institutions in Europe, Milton Keynes International Centre for Distance Learning/ Londres, 1997, 126 p., index.

Bibliographie établie dans le cadre du programme européen ManageLearn (Socrates) par un ensemble de partenaires européens, à l'intention des institutions de l'enseignement supérieur souhaitant établir ou développer un programme d'enseignement à distance.

5 LEMOINE Marie-Hélène, La formation à distance: dossier documentaire, CIEP, Service de documentation, 1999/02, dossier 154 p. + disquette.

Ce dossier offre quatre entrées: quelques définitions, une bibliographie analytique (ingénierie de la FOAD, études de cas, autoformation, avec des extraits), des adresses et une sélection commentée de soixante sites internet (avec également des extraits significatifs). Bientôt disponible sur le site web du CIEP « http://www.ciep.fr/doc/ ».

ORAVEP : Observatoire des ressources pour la formation/Paris, Éducation et formation Sur les réseaux: rôles joués par les collectivités locales et par le système éducatif, OTV : Observatoire des télécommunications dans la ville/Paris, Les publications de l'Observatoire, 1998/01, $73 \mathrm{p}$.

Les collectivités locales sont de plus en plus concernées et sollicitées par l'entrée dans les établissements scolaires et les organismes de formation d'adultes des technologies de l'information. Cette étude fournit un état des lieux, montre la complexité des rapports entre les acteurs et met en évidence quelques politiques parmi les mieux accomplies en région.

7 PERRIAULT Jacques, La communication du savoir à distance, L'Harmattan/Paris, Éducation et formation. Série références, 1996, 255 p., bibliogr.

L'enseignement à distance est à l'origine d'une des plus grandes mutations qu'ait connue l'éducation mondiale, encore passée quasiment inaperçue. Cet ouvrage à caractère prospectif fait le point sur les évolutions des dix dernières années, met en lumière la politique européenne et les grandes orientations mondiales, et s'achève en indiquant comment la société civile et l'industrie peuvent aider l'éducation à relever les défis.

PORTER Lynnette R., Creating the virtual classroom: distance learning with the Internet, John Wiley \& Sons/New-York/États-Unis, 1997, 260 p., bibliogr., glossaire, index.

Cet ouvrage fournit des conseils pour la préparation, la gestion et le financement d'un programme d'enseignement à distance sur internet. Après avoir détaillé les différents outils et techniques utilisables, il aborde les questions de l'évaluation et de la promotion du programme. On trouvera en annexe des ressources en ligne utiles aux concepteurs de programmes d'enseignement à distance (sites web, listes de diffusions, revues électroniques, etc.). 


\section{L'éducation à distance au service de l'aide au développement}

BOUSQUET Antoine, Éducation et formation dans l'Union européenne: un espace de coopération, Documentation française/Paris « Réflexe Europe », 1998, 154 p.

La nécessité pour la Communauté économique européenne d'intervenir dans le secteur de l'éducation s'est imposée rapidement comme une réponse au désir politique de réaliser une "véritable communauté culturelle»; de 1974 à 1993, le projet communautaire s'élabore progressivement et, depuis 1993, l'éducation et la formation font partie intégrante de l'action communautaire. Mais la Commission souhaite aller plus loin; en témoignent le Livre blanc Enseigner et apprendre... et le rapport Accomplir l'Europe par l'éducation et la formation. Cet ouvrage fait le point sur la façon dont la Communauté a acquis cette compétence «éducation-formation » puis sur les modalités d'organisation de la coopération communautaire dans ce domaine.

CIFFAD : Consortium francophone international de formation à distance, Séminaire international sur la formation à distance. Conakry, 18-23 septembre 1995, rapport général, ACCT, École internationale de Bordeaux/Talence/France, 1995, 67 p. +27 p.

Regroupant des participants des pays du Nord et du Sud, ce séminaire étudie la situation de la formation à distance dans les pays représentés, l'apport des nouvelles technologies de la communication et de l'information, les projets à retenir et leurs modalités de mise en œuvre, les partenariats et les réseaux à consolider.

11 FARNES Nicholas, New structures to reform higher education in Central and Eastern Europe: the role of distance education, European journal of education, vol. 32, $n^{\circ} 4,1997 / 12$, p. 379-398.

L'article fait le point sur l'importance de l'enseignement à distance dans les réformes de l'enseignement supérieur des pays d'Europe de l'Est, en particulier la Hongrie. L'accent est mis notamment sur les programmes financés par la Communauté européenne.

THOMAS Régine, Formation à distance au niveau de l'enseignement supérieur dans les pays d'Afrique francophone et lusophone, secrétariat d'État à la coopération/Paris, 1997/08, 154 p. Cette enquête comporte une introduction, les fiches descriptives de l'état de la situation dans vingt-quatre pays d'Afrique (sources, repères, statistiques, place de l'internet), et trois études de cas plus poussées pour la Côte d'Ivoire, Djibouti ou Madagascar, en raison du caractère significatif et exemplaire des expériences qui y sont menées. En annexe, le questionnaire d'enquête et les actions menées dans le cadre de la coopération multilatérale (Agence pour la francophonie, AUPELF-UREF). Ce dossier, évolutif, est destiné à évoluer en ligne au fur à mesure des mises à jour, "http:// www.lid.jussieu.fr/resafad/observatoire/index.html ».

\section{L'éducation à distance et la formation tout au long de la vie}

13 Actes du séminaire sur la formation à distance des cadres dans les pays du pourtour méditerranéen, Commission française pour Unesco, Commission marocaine pour l'Unesco, 1996, $381 \mathrm{p}$.

La problématique de la $\mathrm{FAD}$ est abordée par thèmes puis par pays. 
CASPAR Pierre (dir.), Nouvelles technologies éducatives et réseaux de formation: des entreprises parlent de leur expérience, Éd. d'Organisation/Paris, 1998, 241 p., index, bibliogr.

Quatre entreprises françaises (EDF, Gaz de France, France Télécom et Renault) évoquent leurs recherches récentes sur les contributions de la formation professionnelle dans la résolution de leurs problèmes, en particulier des dispositifs de formation ouverte et à distance.

Distance education for primary school teachers, Asian Development Bank/Manila/ Philippines, 1997, $416 \mathrm{p}$.

Études générales sur la formation à distance des enseignants du primaire (tendances, méthodes, technologies, coûts) et dispositifs nationaux pour cinq pays d'Asie: le Bangladesh, la République populaire de Chine, l'Inde, le Pakistan et l'Indonésie.

LEPLATRE Françoise (coord.), Formations ouvertes et à distance: enjeux et perspectives, pratiques et expériences, Actualité de la formation permanente, $\mathrm{n}^{\circ} 156$ et 157, 1998/10 et 12, p. 37-91 et p. 25-91.

17 Dossier sur l'emploi des nouvelles technologies dans la formation des maîtres, European journal of teacher education, vol. 20, $\mathrm{n}^{\circ} 1,1997,108$ p., bibliogr.

Bilan de l'éducation ouverte et à distance et de l'emploi des nouvelles technologies dans la formation des enseignants des pays de l'Union européenne.

\section{Aspects pédagogiques et sociologiques de l'éducation à distance}

BATES Peter J., Telematics for flexible and distance learning: final report, Commission européenne DG XIII/Bruxelles/Belgique, 1996, 104 p.

Présentation des résultats de trente projets, menés dans le cadre du programme Delta, regroupés autour de trois thèmes majeurs : amélioration de l'accès aux ressources d'enseignement; rencontre de la demande croissante de solutions d'enseignement rentables; amélioration de la qualité, définition de standards, compréhension et stimulation du marché.

BERNARD Michel, Penser la mise à distance en formation, L'Harmattan/Paris, Éducation et formation, 1999, 298 p., bibliogr., tabl, lexique, graph.

La formation à distance et la formation ouverte et à distance sont considérées comme des étapes transitoires. La "mise à distance en formation" se propose de traiter la distance dans sa complexité afin de contribuer à l'émergence d'un nouvel esprit formatif avec des pratiques nouvelles.

20 CLEMENT Danièle, Quel type de médiation dans l'apprentissage coopératif à distance?, Spirales, $\mathrm{n}^{\circ} 17,1996, \mathrm{p} .117-126$, bibliogr.

L'apprentissage à distance suppose de mettre en œuvre deux types de médiation: humaine, dans la création d'un lien entre les apprenants, et technique. Cet article repose sur la présentation du projet « co-learn » de l'université de Lille I.

COSTE Daniel (coord.), Enseignement et formation à distance, Études de linguistique appliquée, 1999/01, p. 5-128.

Numéro consacré à l'enseignement à distance des langues en tant que révélateur de mouvements, de tensions et d'enjeux d'une portée très large. Cet enseignement ne repose pas seulement sur le conditionnement industriel du savoir multiplié 
uniformément pour tous les consommateurs potentiels. La banalisation du numérique fait prévaloir l'excès plutôt que le manque de ressources langagières. Les modes de communication et les réseaux d'interaction se trouvent démultipliés et diversifiés. Les échanges ne se limitent plus à la relation entre l'étudiant et l'enseignant: les apprenants ont finalement à affronter de nouvelles instances d'évaluation, officielles ou pas. Ces questions sont développées à partir d'un tour d'horizon des dispositifs développés en Amérique du nord et en France (CNED, méthode Vifax, méthode Tandem, université de Rouen).

D'HALLUIN C, La présence à distance c'est possible ou d'un bon usage des médias, in Entretiens internationaux sur l'EAD, CNED/Paris, 1995/10, p. 107-115.

QUERE Maryse, Vers un enseignement supérieur sur mesure, 1996, 20 p.

Ce rapport soumet à la communauté universitaire les questions relatives aux procédures actuelles (qualité, dérives), la relation enseignant-étudiant, l'évaluation, les publics à effectifs réduits, les antennes, le statut des étudiants à distance, les questions liées à la production d'outils, le financement, le dialogue avec les régions, la recherche, la formation des maîtres. Il présente également les acteurs et les dispositifs qui concourent à l'EAD.

STERQUEL Jean-François, La formation à distance en français langue étrangère: quelques propositions actuelles des universités françaises tournées vers l'international, 1995/06, 40 p., bibliogr., annexes.

Ce dossier présente sous forme de fiches techniques les principaux opérateurs français pour la formation à distance en français langue étrangère, et son originalité consiste à offrir les résultats d'une enquête menée auprès des étudiants étrangers inscrits à ces formations, ainsi que des entretiens avec des spécialistes.

Thot : Nouvelles de la formation à distance en francophonie.

Revue électronique consultable sur : http://thot.cursus.edu/

\section{AUTEUR}

\section{MARIE LAKERMANCE}

Documentaliste au CIEP 